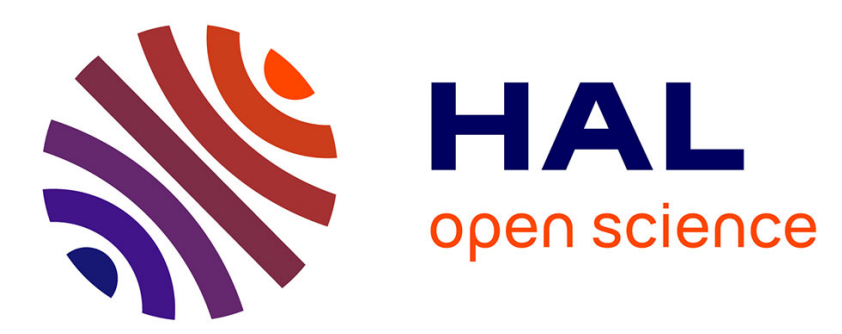

\title{
Raman scattering investigation of the boson peak in a sodium silicate glass
}

\author{
Giacomo Baldi, Aldo Fontana, F. Rossi, Giulio Monaco
}

\section{To cite this version:}

Giacomo Baldi, Aldo Fontana, F. Rossi, Giulio Monaco. Raman scattering investigation of the boson peak in a sodium silicate glass. Philosophical Magazine, 2010, pp.1. 10.1080/14786435.2010.523020 . hal-00640220

\section{HAL Id: hal-00640220 \\ https://hal.science/hal-00640220}

Submitted on 11 Nov 2011

HAL is a multi-disciplinary open access archive for the deposit and dissemination of scientific research documents, whether they are published or not. The documents may come from teaching and research institutions in France or abroad, or from public or private research centers.
L'archive ouverte pluridisciplinaire HAL, est destinée au dépôt et à la diffusion de documents scientifiques de niveau recherche, publiés ou non, émanant des établissements d'enseignement et de recherche français ou étrangers, des laboratoires publics ou privés. 


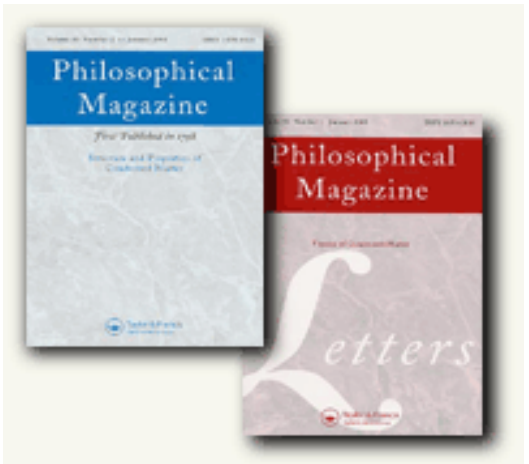

\section{Raman scattering investigation of the boson peak in a sodium silicate glass}

\begin{tabular}{|r|l|}
\hline Journal: & Philosophical Magazine \& Philosophical Magazine Letters \\
\hline Manuscript ID: & TPHM-10-Jun-0256.R1 \\
\hline Journal Selection: & Philosophical Magazine \\
\hline Date Submitted by the & 02-Sep-2010 \\
\hline Complete List of Authors: & $\begin{array}{l}\text { Baldi, Giacomo; European Synchrotron Radiation Facility } \\
\text { Fontana, Aldo; university of trento, physics } \\
\text { Rossi, F.; Universita' di Trento, Fisica } \\
\text { Monaco, Giulio; ESRF }\end{array}$ \\
\hline Keywords: & boson peak, glass, elasticity \\
\hline Keywords (user supplied): & \multicolumn{2}{|r}{} \\
\hline
\end{tabular}

\section{SCHOLARONE \\ Manuscripts}




\title{
Raman scattering investigation of the boson peak in a sodium silicate glass
}

\section{Giacomo Baldi}

IPCF-CNR, UOS di Roma, 00185 Roma, Italy and

European Synchrotron Radiation Facility, BP220, 38043 Grenoble, France

giacomo.baldi@esrf.fr

\author{
Aldo Fontana \\ Physics Department, Trento University, 38050 Povo, Trento, Italy and \\ IPCF-CNR, UOS di Roma, 00185 Roma, Italy
}

Flavio Rossi

Physics Department, Trento University, 38050 Povo, Trento, Italy

Giulio Monaco

European Synchrotron Radiation Facility, BP220, 38043 Grenoble, France

(Received; final version received)

\begin{abstract}
Raman scattering is used to investigate the vibrational spectrum of a sodium silicate glass around the boson peak frequency range at temperatures between 10 and $450 \mathrm{~K}$. The comparison with the density of states measured with coherent neutron scattering allows one to determine the Raman coupling function. This quantity presents a linear frequency dependence in the boson peak region and a negligible temperature dependence, confirming similar observations in other glasses. The Raman spectra at different temperatures scale according to the Debye frequency determined from the sound velocity measured at terahertz frequency with inelastic x-ray scattering. This result confirms previous neutron scattering data and the importance of measuring the elastic properties of the glass in the proper frequency range.
\end{abstract}

Keywords: boson peak; glass; elasticity.

\section{Introduction}

Topologically disordered solids are characterized by the lack of long range structural order. In spite of the absence of periodicity, these systems can sustain the propagation of sound up to terahertz frequencies [1]. However their vibrational dynamics is strongly affected by the presence of disorder. Particularly evident is the appearance of 
an excess of vibrational modes over the Debye level at terahertz frequencies, usually called the boson peak (BP).

The boson peak variation as a function of an external control parameter has been deeply investigated in the last two decades. In particular the BP evolution has been studied as a function of temperature $[1,2,3]$, pressure $[4,5]$ or density $[6,7,8]$, varying the sample preparation [9] and during chemical vitrification [10]. An increase in sample density is usually accompanied by a shift of the BP towards higher frequencies associated to a reduction of its intensity. In the case of vitreous silica the same behavior is observed as the temperature is increased [2,3].

A major question is whether the BP shift can be explained by corresponding variations in the macroscopic elastic medium properties, such as density and sound speeds. Using nuclear inelastic scattering Monaco et al. [6] have shown that in permanently densified samples the BP shift follows the variation of the Debye frequency $v_{D}$, in the range of densities where local structure modifications can be neglected. A similar result is obtained in recent numerical simulations of systems with a varying degree of fragility [11]. On the contrary a pressure dependence of the BP frequency stronger than the $v_{D}$ variation has been reported by Niss et al. [4] in studies of polymeric glasses.

In a recent work [12] we have shown that the comparison of the BP variations to those of the macroscopic continuum medium requires a careful determination of the elastic properties of the glass. Specifically the sound velocity, which is used to estimate the Debye frequency, needs to be measured in a frequency range where contributions from anharmonicity or relaxation processes can be neglected. This can be accomplished by measuring the sound velocity at high frequency, in the terahertz range, by means of inelastic $x$-rays scattering. 
In this paper we present new data on the vibrational spectrum of a sodium silicate glass in the BP range, measured with Raman scattering at temperatures between 10 and $450 \mathrm{~K}$, in the glassy state. The comparison of these new data with neutron scattering measurements allows us to determine the Raman coupling function, which shows an almost linear frequency dependence and a negligible temperature dependence. The Raman data give further support to the importance of measuring the elastic properties of the medium in the proper frequency range.

\section{Experimental}

The silicate glass sample, $60 \% \mathrm{SiO}_{2}-40 \% \mathrm{Na}_{2} \mathrm{O}$, has been prepared using the standard melt-quenching technique. Pure $\mathrm{SiO}_{2}$ and $\mathrm{Na}_{2} \mathrm{CO}_{3}$ powders have been melted at 1600 ${ }^{\circ} \mathrm{C}$ in air in a Pt crucible. The obtained sample has been annealed for $6 \mathrm{~h}$ at the glass transition temperature $\left(\mathrm{T}_{\mathrm{g}} \sim 700 \mathrm{~K}[13]\right)$. A cubic shaped sample of dimensions $5 \mathrm{x} 4 \mathrm{x} 4$ $\mathrm{mm}^{3}$ has been cut and optically polished. The inclusion of $\mathrm{Na}$ in the $\mathrm{SiO}_{2}$ network has the effect of inducing a strong reduction of the glass transition temperature and an increase of the fragility of the system $(\mathrm{m} \sim 50[14])$.

The elastic properties of the glass have been studied by means of Brillouin light (BLS) and x-ray (IXS) scattering as a function of temperature, as previously reported in ref. [12]. The BLS signal in right angle geometry has been measured using a Sopra DPDM monochromator. The spectrometer employs an incident wavelength of $5145 \AA$ and is operated in single pass configuration at the 11th order, providing a resolution of $1.2 \mathrm{GHz}$. The right angle geometry allows one to measure both the longitudinal and the transverse sound velocities. The value of the longitudinal sound velocity around room temperature has been checked in backscattering geometry. For this test we employed a Sandercock type (3+3)-pass tandem Fabry-Perot 
interferometer. The spectrometer works with an incident wavelength of $532 \mathrm{~nm}$ and has an energy resolution $\sim 0.6 \mathrm{GHz}$. The sound velocity in the $\mathrm{THz}$ regime has been measured by means of IXS. The experiment has been performed at the beamline ID16 of the European Synchrotron Radiation Facility. An incident beam of $23.7 \mathrm{keV}$ energy is monochromatized by the $(12,12,12)$ reflection of a Si crystal, providing an energy resolution of $1.4 \mathrm{meV}$. The sound velocity has been measured at a small exchanged wavevector, $\mathrm{q}=0.12 \AA^{-1}$.

Information on the density of vibrational states is obtained by means of Raman and inelastic neutron scattering experiments. The Raman measurements in HV geometry were performed using a standard experimental set-up Jobin Yvon U1000. The spectra were measured at temperatures in the range from $10 \mathrm{~K}$ to $450 \mathrm{~K}$. The intensity of the measured spectra is plotted as a function of frequency in figure 1 . The curves are normalized to the high frequency peaks, outside the frequency range shown in the figure. The spectra show a prominent boson peak at a frequency $v \sim 1.6 \mathrm{THz}$, best visible at high temperatures. The neutron scattering experiment has been performed at the IN4 spectrometer of the Institut Laue Langevin in Grenoble. Details on the experimental setup and data analysis can be found in ref. [12].

\section{Discussion}

\section{The Raman coupling function}

The Raman intensity, $I_{R}$, is conveniently written in terms of the reduced Raman intensity $\mathrm{I}^{\text {red }}$ :

$$
I^{r e d}(v, T)=\frac{I_{R}}{v[n(v, T)+1]}
$$




$$
I^{r e d}(v, T)=\frac{C(v, T) g(v)}{v^{2}}
$$

where $C(v, T)$ is a function accounting for the light-vibration coupling.

The neutron inelastic scattering technique allows to obtain a reliable determination of the density of vibrational states $g(v)$, as explained in ref. [12]. The comparison between the Raman and the neutrons spectra thus allows to determine the Raman coupling function $C(v, T)$, which is plotted in figure 2. The coupling function is a smooth function of frequency and it increases approximately linearly with frequency in the boson peak range. Moreover its shape is not affected by temperature, whose effect is only a slight shift of $C(v, T)$ to higher values as $\mathrm{T}$ is increased.

\section{Elastic properties and the scaling of the boson peak}

The comparison between the BLS and IXS data allows one to investigate the temperature evolution of the elastic properties of the system in different frequency ranges, some tens of GHz for the BLS experiment and a few $\mathrm{THz}$ for the IXS one. The temperature dependence of the sound velocity, both longitudinal and transverse, normalized to its low temperature value, is plotted in figure 3 . The transverse sound velocity at terahertz frequency cannot be directly measured because the IXS technique probes the longitudinal current correlation function. The plotted values are estimated exploiting the Cauchy relation: $M=A+B G$, where $M=\rho \mathrm{v}_{L}^{2}$ and $G=\rho \mathrm{v}_{T}^{2}$ are the longitudinal and the shear modulus respectively and $\rho$ is the mass density. This 
equation has been shown to hold for a variety of materials at every frequency, with $B \sim 3$ and $A$ a system dependent quantity [15]. The parameter $A$ can be estimated from the BLS data, which correctly obey the Cauchy relation [12].

Figure 3 shows that the sound velocity in the GHz frequency range is strongly temperature dependent and thus affected by anharmonicity or relaxation processes that tend to reduce the elastic modulus at high temperature. On the contrary, in the $\mathrm{THz}$ regime the sound velocity appears to be temperature independent within the experimental accuracy. This observation is of great relevance when the temperature or pressure dependence of the boson peak is compared to the corresponding evolution of the elastic properties of the medium. This comparison can be done estimating the Debye frequency:

$$
v_{D}=\frac{\mathrm{q}_{D} \mathrm{v}_{D}}{2 \pi}=\sqrt[3]{\frac{3}{4 \pi} \frac{\rho(T)}{m}} \mathrm{v}_{D}(T)
$$

where $\mathrm{q}_{\mathrm{D}}$ and $\mathrm{v}_{\mathrm{D}}$ are the Debye wavevector and sound velocity respectively and $m$ is the average atomic mass. In the temperature range here investigated, below the glass transition temperature, the density of the sodium silicate glass doesn't change appreciably. For this reason the temperature dependence of the Debye frequency is entirely due to the variation of the sound velocity.

On general grounds the sound velocity can depend on temperature because of the presence of relaxation processes or anharmonicity. Concerning the contribution of anharmonic terms we can distinguish between a quasi-harmonic part and a "purely" anharmonic contribution. The quasi-harmonic term may induce a variation of the sound velocity because the elastic constants of the glass rearrange to different values when the temperature is changed. This contribution is expected to be frequency independent. With "purely" anharmonic contribution we indicate the effect of terms in 
the expansion of the inter-atomic potential which are of third and higher order in the atomic displacement around the equilibrium configuration. This contribution is frequency dependent and gives rise to a temperature dependence of the sound velocity and to damping of the sound waves [17].

Because of the frequency dependence of the sound velocity we may conclude that also the Debye frequency is frequency dependent. However here the Debye frequency is used to estimate the temperature evolution of the elastic properties of the continuum medium. We should thus measure it in a frequency range where it is not affected by "purely" anharmonic terms or by relaxation processes. This is accomplished by measuring it in the terahertz frequency range. It is worth noting that the sound velocity may still be temperature dependent in the terahertz range, because of the presence of a quasi-harmonic mechanism, due, for instance, to a structural rearrangement of the system with temperature or to a temperature dependence of the density. This is not the case in the silicate glass here studied, as shown by the temperature independence of the sound velocity determined with IXS.

Once the Debye frequency is known, one can rescale the measured density of vibrational states, $g(v)$, plotting it as a function of the quantity $v / v_{D}$. In order to maintain the correct normalization, the density of states in the new variable is: $\tilde{g}\left(v / v_{D}\right)=g(v) \cdot v_{D}$. The reduced density of vibrational states, $g(v) / v^{2}$, is plotted in figure 4 as a function of the variable $v / v_{D}$. The figure shows a comparison between the Raman (top) and inelastic neutron scattering (bottom) data. In the left column the Debye frequency calculated from the IXS sound velocity, $v_{D}^{I X S}$, is used to scale the figure axis. The Raman intensity is scaled assuming that the coupling function, $C(v, T)$, is linear in frequency, as confirmed by the comparison with the neutron data (see figure 2). The left column of figure 4 shows that the boson peak in 
this silicate glass is temperature independent in the temperature range here explored. This is confirmed by both the Raman and the neutron data. Also the elastic properties of the continuum medium are not affected by temperature, and they are properly described by $v_{D}^{I X S}$. A temperature dependence of the reduced density of states can be seen below the boson peak frequency, in both the Raman and neutron data. This quasi-elastic contribution is probably associated to the same anharmonic or relaxation processes that affect the sound velocity at $\mathrm{GHz}$ frequencies.

On the right column of figure 4 the scaling is performed using the Debye frequency determined from the BLS data, $v_{D}^{B L S}$. The temperature dependence of this quantity induces a spurious variation of the scaled reduced density of states, appreciable in both the Raman and the neutrons data. The figure allows to better appreciate the importance of using sound velocity values which are not affected by relaxation or anharmonic processes, when these are employed to estimate the elastic properties of the continuum medium.

\section{Conclusions}

The investigation of the vibrational dynamics of a sodium silicate glass by means of Raman scattering further supports the conclusions obtained with inelastic neutron scattering. The comparison of the spectra obtained with the two techniques allows us to determine the Raman coupling function, which is marked by a linear frequency dependence in the boson peak region. This result is used to estimate the reduced vibrational density of states from the Raman intensity and to scale it with the Debye frequency. This quantity is independently determined from the sound velocity obtained with BLS and IXS. 
A proper scaling of the boson peak is obtained using the IXS values of the sound velocity, which are not affected by anharmonicity or relaxation processes. This conclusion is confirmed by both neutrons and Raman scattering. The present contribution indicates the importance of measuring the elastic properties of the glass in the proper frequency range if a characterization of the elastic continuum medium is desired.

References:

[1] F. Sette, M.H. Krisch, C. Masciovecchio, G. Ruocco and G. Monaco, Science 280 (1998) p.1550;

C. Masciovecchio et al., Phil. Mag. B 79 (1999) p. 2013;

B. Ruzicka et al., Phys. Rev. B 69 (2004) p.100201(R);

O. Pilla et al., J. Phys.: Condens. Matter 16 (2004) p.8519;

G. Baldi et al., Phys. Rev. B 77 (2008) p.214309.

[2] A. Wischnewski, U. Buchenau, A.J. Dianoux, W.A. Kamitakahara and J.L. Zarestky, Phys. Rev. B 57 (1998) p.2663.

[3] S. Caponi, A. Fontana, F. Rossi, G. Baldi and E. Fabiani, Phys. Rev. B 76 (2007) p.092201.

[4] K. Niss et al., Phys. Rev. Lett. 99 (2007) p.055502.

[5] L. Hong et al., Phys. Rev. B 78 (2008) p.134201.

[6] A. Monaco et al., Phys. Rev. Lett. 97 (2006) p.135501.

[7] L. Orsingher et al., J. Chem. Phys. 132 (2010) p.124508.

[8] M. Zanatta, G. Baldi, S. Caponi et al., Phys. Rev. B, accepted for publication.

[9] A. Monaco et al., Phys. Rev. Lett. 96 (2006) p.205502.

[10] S. Caponi et al., Phys. Rev. Lett. 102 (2009) p.027402.

[11] H. Shintani and H. Tanaka, Nature Materials 7 (2008) p.870.

[12] G. Baldi, A. Fontana, G. Monaco, L. Orsingher, S. Rols, F. Rossi and B. Ruta, Phys. Rev. Lett. 102 (2009) p.195502.

[13] S. Suzuki and Y. Abe, J. Non-Cryst. Solids 43 (1981) p.141.

[14] R. Bohmer et al., J. Chem. Phys. 99 (1993) p.4201.

[15] D. Fioretto et al., J. Chem. Phys. 128 (2008) p.214502.

[16] G. Baldi, V. M. Giordano, G. Monaco and B. Ruta, Phys. Rev. Lett. 104 (2010) p.195501.

[17] A. Akhiezer, J. Phys. (USSR) 1 (1939) p.277.

Figure captions:

Figure 1 (color online). Raman intensity as a function of frequency at the indicated temperatures (the inelastic signal increases with temperature).

Figure 2 (color online). Raman coupling function as a function of frequency at the indicated temperatures. The line is a guide for the eye showing a linear dependence. 
Figure 3 (color online). Temperature dependence of the longitudinal (panel A)) and transverse (panel B)) sound velocities, normalized to their low temperature values. Squares: IXS data, circles: BLS data in right angle geometry. Triangle: BLS point measured in back scattering. The low temperature sound velocity values for the BLS and IXS data differ because of the negative dispersion effect $[12,16]$. They have been determined from a linear fit of the temperature dependence to be:

$\mathrm{v}_{L, 0}^{B L S}=(5784 \pm 27) \mathrm{m} \mathrm{s}^{-1}, \mathrm{v}_{T, 0}^{B L S}=(3280 \pm 35) \mathrm{m} \mathrm{s}^{-1}, \mathrm{v}_{L, 0}^{I X S}=(5454 \pm 26) \mathrm{m} \mathrm{s}^{-1}$, $\mathrm{v}_{T, 0}^{I X S}=(3043 \pm 5) \mathrm{m} \mathrm{s}^{-1}$. The IXS transverse sound velocity is not directly measured and it is computed exploiting the Cauchy relation, as explained in the text.

Figure 4 (color online). Left column: upper panel: reduced Raman intensity as a function of $v / v_{D}^{I X S}$; lower panel: reduced density of states measured with inelastic neutron scattering as a function of $v / v_{D}^{I X S}$. Right column: the same quantities of the left column rescaled according to the Debye frequency determined from the BLS sound velocity values, $v_{D}^{B L S}$. 


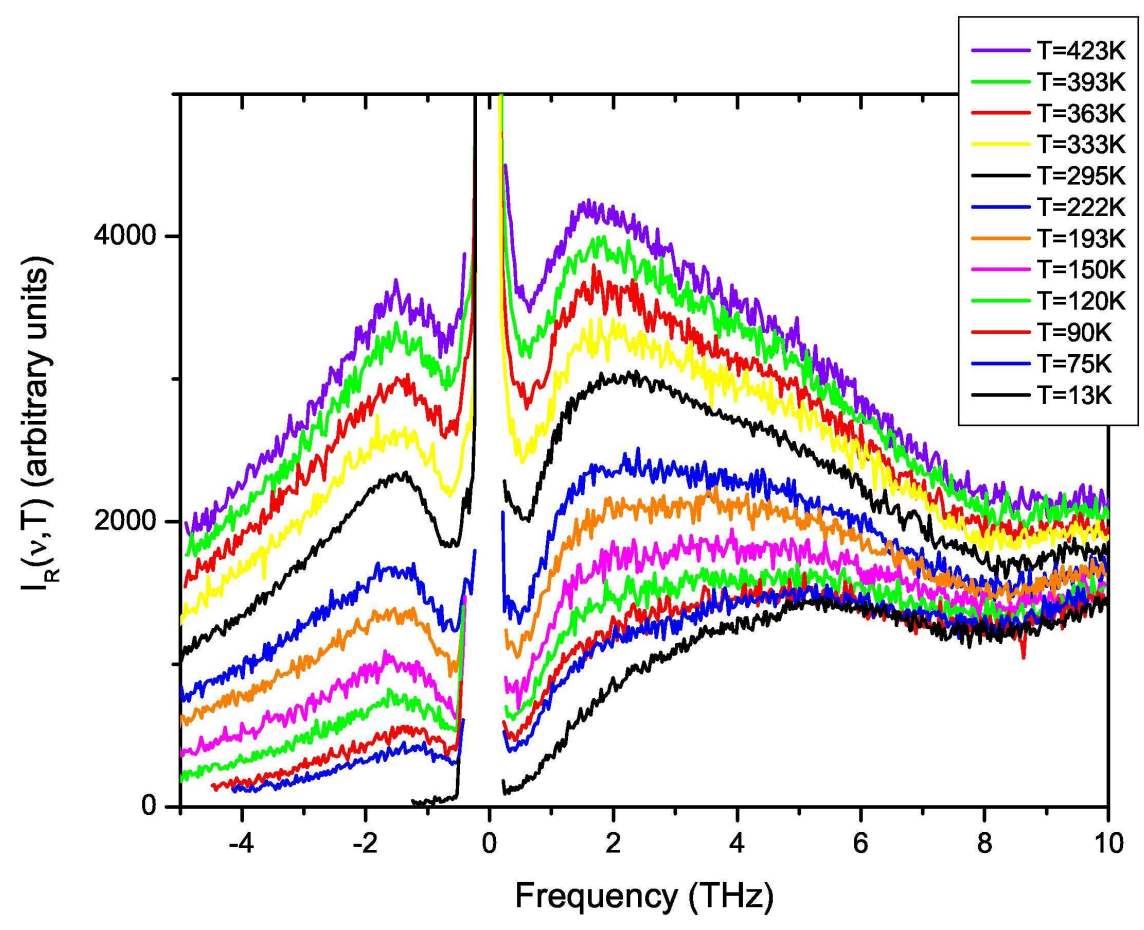

(color online). Raman intensity as a function of frequency at the indicated temperatures (the inelastic signal increases with temperature). $107 \times 85 \mathrm{~mm}(600 \times 600 \mathrm{DPI})$ 


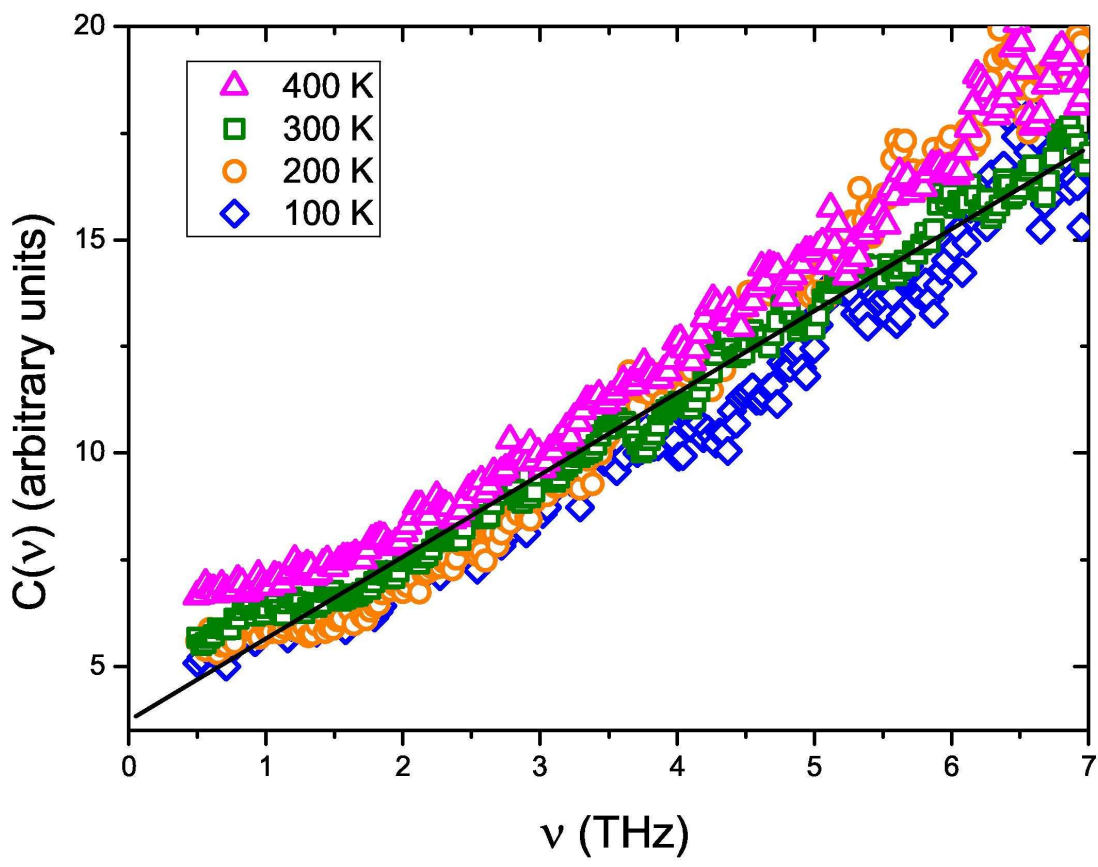

(color online). Raman coupling function as a function of frequency at the indicated temperatures. The line is a guide for the eye showing a linear dependence. $106 \times 86 \mathrm{~mm}(600 \times 600$ DPI $)$ 
(color online). Temperature dependence of the longitudinal (panel A)) and transverse (panel B)) sound velocities, normalized to their low temperature values. Squares: IXS data, circles: BLS data in right angle geometry. Triangle: BLS point measured in back scattering. The low temperature sound velocity values for the BLS and IXS data differ because of the negative dispersion effect $[12,16]$. The IXS transverse sound velocity is not directly measured and it is computed exploiting the Cauchy relation, as explained in the text. $120 \times 86 \mathrm{~mm}(600 \times 600 \mathrm{DPI})$ 

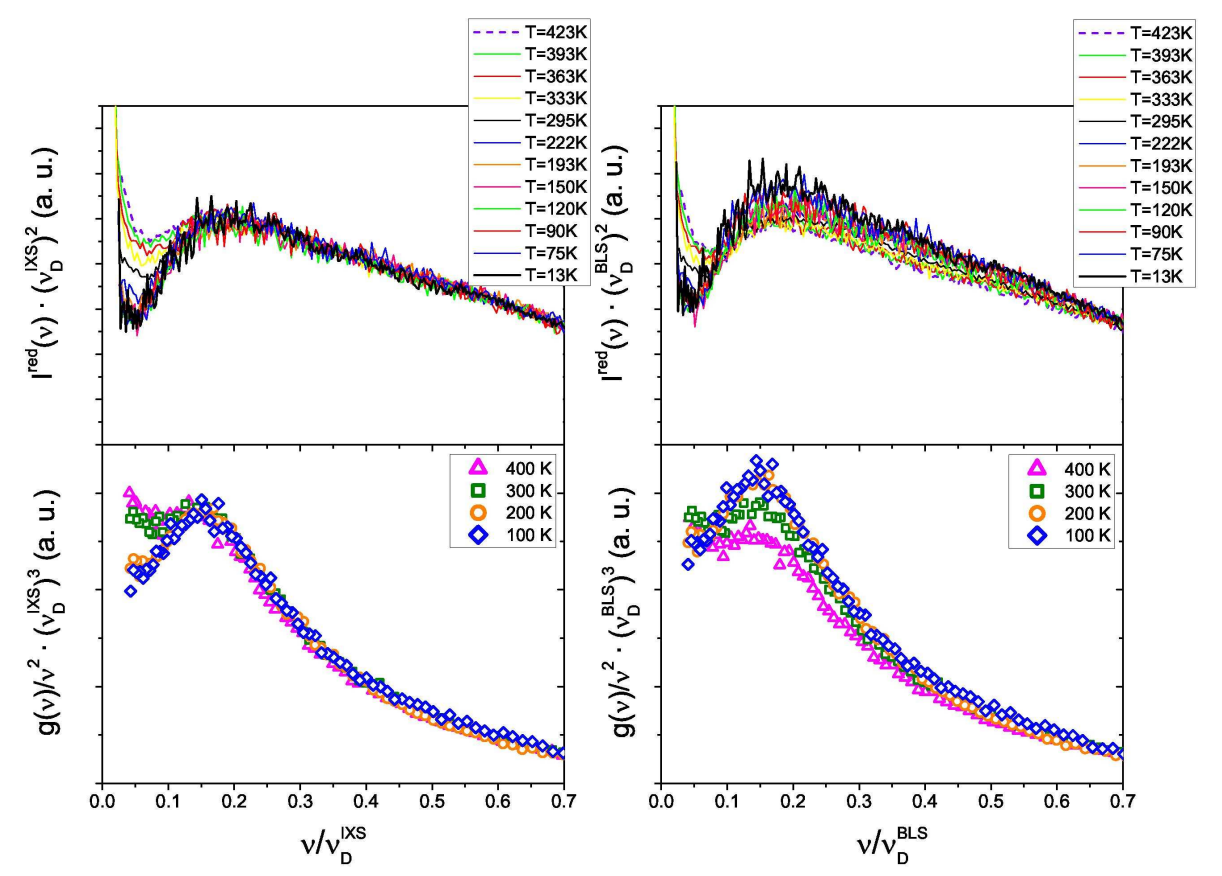

(color online). Left column: upper panel: reduced Raman intensity as a function of frequency in units of the Debye frequency measured by IXS; lower panel: reduced density of states measured with inelastic neutron scattering. Right column: the same quantities of the left column rescaled according to the Debye frequency determined from the BLS sound velocity values. $123 \times 92 \mathrm{~mm}(600 \times 600 \mathrm{DPI})$ 